Low-dose megavoltage cone-beam CT imaging using thick, segmented scintillators

This article has been downloaded from IOPscience. Please scroll down to see the full text article.

2011 Phys. Med. Biol. 561509

(http://iopscience.iop.org/0031-9155/56/6/001)

View the table of contents for this issue, or go to the journal homepage for more

Download details:

IP Address: 141.211.173.82

The article was downloaded on 06/04/2012 at 16:54

Please note that terms and conditions apply. 


\title{
Low-dose megavoltage cone-beam CT imaging using thick, segmented scintillators
}

\author{
Youcef El-Mohri ${ }^{1}$, Larry E Antonuk, Qihua Zhao, \\ Richard B Choroszucha, Hao Jiang and Langechuan Liu \\ Department of Radiation Oncology, University of Michigan, Ann Arbor, MI 48109, USA \\ E-mail: elmohri@umich.edu
}

Received 18 October 2010, in final form 6 January 2011

Published 16 February 2011

Online at stacks.iop.org/PMB/56/1509

\begin{abstract}
Megavoltage, cone-beam computed tomography (MV CBCT) employing an electronic portal imaging device (EPID) is a highly promising technique for providing soft-tissue visualization in image-guided radiotherapy. However, current EPIDs based on active matrix flat-panel imagers (AMFPIs), which are regarded as the gold standard for portal imaging and referred to as conventional MV AMFPIs, require high radiation doses to achieve this goal due to poor $\mathrm{x}$-ray detection efficiency ( $\sim 2 \%$ at $6 \mathrm{MV})$. To overcome this limitation, the incorporation of thick, segmented, crystalline scintillators, as a replacement for the phosphor screens used in these AMFPIs, has been shown to significantly improve the detective quantum efficiency (DQE) performance, leading to improved image quality for projection imaging at low dose. Toward the realization of practical AMFPIs capable of low dose, soft-tissue visualization using MV CBCT imaging, two prototype AMFPIs incorporating segmented scintillators with $\sim 11 \mathrm{~mm}$ thick CsI:Tl and $\mathrm{Bi}_{4} \mathrm{Ge}_{3} \mathrm{O}_{12}$ (BGO) crystals were evaluated. Each scintillator consists of $120 \times 60$ crystalline elements separated by reflective septal walls, with an element-to-element pitch of $1.016 \mathrm{~mm}$. The prototypes were evaluated using a bench-top CBCT system, allowing the acquisition of 180 projection, $360^{\circ}$ tomographic scans with a $6 \mathrm{MV}$ radiotherapy photon beam. Reconstructed images of a spatial resolution phantom, as well as of a water-equivalent phantom, embedded with tissue equivalent objects having electron densities (relative to water) varying from $\sim 0.28$ to $\sim 1.70$, were obtained down to one beam pulse per projection image, corresponding to a scan dose of $\sim 4 \mathrm{cGy}-\mathrm{a}$ dose similar to that required for a single portal image obtained from a conventional MV AMFPI. By virtue of their significantly improved DQE, the prototypes provided low contrast visualization, allowing
\end{abstract}

1 Author to whom any correspondence should be addressed. 
clear delineation of an object with an electron density difference of $\sim 2.76 \%$. Results of contrast, noise and contrast-to-noise ratio are presented as a function of dose and compared to those from a conventional MV AMFPI.

(Some figures in this article are in colour only in the electronic version)

\section{Introduction}

Over the past several years, many imaging techniques have been developed to facilitate image-guided radiation therapy (IGRT) (Saw et al 2006, Dawson and Jaffray 2007) with the goal of achieving increased radiation dose to tumor volumes while minimizing dose to surrounding normal tissues and critical structures. These techniques generally provide in-room, 3D volumetric image information and therefore are considered complementary to portal imaging, which is typically achieved using an electronic portal imaging device (EPID). Among the advantages of $3 \mathrm{D}$ imaging is the possibility of not only visualizing the bony anatomy, which typically is used as a surrogate for tumor localization (and often visible in EPID images), but also soft-tissue structures, allowing assessment of anatomical changes over the course of radiation treatment (Barker et al 2004). One type of 3D imaging technique involves the acquisition of computed tomography (CT) images using a diagnostic CT scanner, referred to as 'CT on rails' (Court et al 2003). In this technique, the treatment couch is moved between the treatment gantry and the $\mathrm{CT}$ scanner. Another technique involves the acquisition of cone-beam computed tomography (CBCT) images using a kilovoltage $(\mathrm{kV}) \mathrm{x}$-ray source and an active matrix flat-panel imager (AMFPI), both mounted orthogonally to the treatment gantry (Jaffray and Siewerdsen 2000, Jaffray et al 2002). This technique, which has seen widespread clinical implementation, results in clinical doses on the order of 1-3 cGy (Islam et al 2006, Amer et al 2007)—doses that are approximately equivalent to those resulting from a single portal image.

An alternative imaging technique involves the use of the megavoltage (MV) treatment beam and an EPID to acquire CT scans-eliminating the need for additional x-ray sources or detectors. One example of such a technique involves the use of tomotherapy equipment, which employs a high-pressure xenon gas detector (Mackie et al 1999, Meeks et al 2005). Another example involves the use of a large-area EPID based on an AMFPI, employing a cone-beam geometry (Pouliot et al 2005, Morin et al 2006). Although the intrinsic contrast of human anatomical structures at MV energies is inferior to that at $\mathrm{kV}$ energies (Groh et al 2002), it has been shown that soft tissue can be visualized at MV energies (Groh et al 2002, Ford et al 2002, Keller et al 2002, Ghelmansarai et al 2005). In addition, the use of the therapy beam for imaging presents some distinct advantages. Compared to kVCT, the images obtained using MVCT exhibit reduced streak artifacts, which are due to the presence of high Z materials such as dental fillings and hip prostheses (Pouliot et al 2005, Yin et al 2005). Furthermore, the CT numbers obtained from MVCT may be readily used for treatment planning dose calculations and inhomogeneity corrections-without the need for a conversion table such as used in KVCT (Guan et al 2002, Langen et al 2005, Morin et al 2005). Finally, the fact that MVCT images are obtained from the therapy beam's eye view eliminates any geometrical uncertainties associated with the additional apparatus of the kVCT configuration.

Despite these advantages, the practical implementation of MV CBCT is constrained by the relatively large dose required to achieve clinically useful contrast resolution using current MV AMFPIs, hereafter referred to as conventional MV AMFPIs (Groh et al 2002). This limitation is the result of the relatively low $x$-ray detection efficiency $(\sim 2 \%$ 
at $6 \mathrm{MV}$ ) of the $\mathrm{x}$-ray converter, which consists of a relatively thick $\mathrm{Gd}_{2} \mathrm{O}_{2} \mathrm{~S}$ : Tb phosphor screen coupled to a $\mathrm{Cu}$ plate, leading to a detective quantum efficiency (DQE) of only $\sim 1 \%$ at zero spatial frequency (El-Mohri et al 2001). In order to overcome this limitation, high efficiency x-ray detectors, based on area detection as well as linear scanning arrays, have been widely investigated (Lewis et al 1992, Mosleh-Shirazi et al 1998a, 1998b, Seppi et al 2003, Pang and Rowlands 2004, Sawant et al 2005, 2006, Samant and Gopal 2006, Sillanpaa et al 2006, Monajemi et al 2006a, 2006b, Rathee et al 2006, Wang et al $2009 \mathrm{~b})$. The premise behind these efforts is that a substantial increase in x-ray detection efficiency achieved through increased detector thickness can be realized, while minimizing the spatial spreading of secondary imaging quanta. One example of such an approach involves the use of thick, large-area, segmented crystalline scintillators consisting of 2D matrices of scintillator crystals (e.g., CsI:Tl, $\mathrm{Bi}_{4} \mathrm{Ge}_{3} \mathrm{O}_{12}$ (BGO), $\mathrm{CdWO}_{4}$ and $\mathrm{ZnWO}_{4}$ ) that are separated by optically opaque/reflective septal walls (Mosleh-Shirazi et al 1998b, Seppi et al 2003, Monajemi et al 2004, Sawant et al 2006, Wang et al 2009b). The use of crystalline scintillators, as opposed to the granular phosphors employed in conventional MV AMFPIs, offers the advantage of lower optical Swank noise-enabling the use of thicker detector material with less severe degradation of DQE (Wang et al 2009a). In addition, the use of opaque/reflective septal walls limits the extent of spatial resolution degradation, which would otherwise be significant as a result of the lateral spreading of secondary optical photons within the scintillator. Recent Monte Carlo simulations of radiation and optical transport have shown that segmented CsI:Tl and BGO scintillators up to $40 \mathrm{~mm}$ thick can provide significantly improved DQE values ( 20\% and $42 \%$, respectively, at zero spatial frequency) (Wang et al 2009a). Other Monte Carlo simulations involving radiation transport at $6 \mathrm{MV}$ have been used to investigate the potential of such scintillators to visualize soft-tissue using CBCT at low dose (Wang et al 2008). From this study, contrastto-noise ratio (CNR) results suggest that a $40 \mathrm{~mm}$ thick, segmented CsI:Tl detector could delineate electron density differences of $\sim 2.3 \%$ and $1.3 \%$ at a dose of 1.54 and 3.08 cGy, respectively.

Toward the realization of thick, large-area segmented scintillators, a series of relatively small-area prototypes with thicknesses ranging from $\sim 11$ to $40 \mathrm{~mm}$, employing BGO and CsI:Tl crystals have been fabricated and examined (Sawant et al 2006, Wang et al 2009b). (While thicker scintillators are more desirable for larger increases in x-ray detection efficiency, examination of a range of scintillator thicknesses allows the probing of limitations and parameter dependences.) These prototypes, which have an element-to-element pitch of 1.016 $\mathrm{mm}$, were coupled to a $0.508 \mathrm{~mm}$ pitch AMFPI array and evaluated for projection imaging using a $6 \mathrm{MV}$ photon beam. The prototypes exhibited input-quantum limited operation at the lowest available dose (i.e. 0.022 cGy corresponding to one beam pulse) with DQE values ranging from $\sim 12$ to 25 times that of a conventional MV AMFPI at zero spatial frequency. Spatial resolution, however, was less than optimal, especially for the thicker prototypes, in part due to some degree of light spreading between adjacent elements as well as less-than-ideal registration between the elements and the AMFPI array pixels (Sawant et al 2006, Wang et al 2009b). In this paper, two of these prototypes, based on BGO and CsI:Tl scintillators and having a thickness of $\sim 11 \mathrm{~mm}$, are examined for MV CBCT imaging using a high resolution ( $0.127 \mathrm{~mm}$ pitch) AMFPI array to circumvent the difficult task of registration. Reconstructed images of tissue-equivalent objects embedded in a water-equivalent phantom are obtained down to the lowest available dose per image frame corresponding to a total scan dose of $\sim 4$ cGy. Performance, in terms of contrast, noise and CNR of the tissue-equivalent objects is examined and compared to that from a prototype imager representative of conventional MV AMFPIs (El-Mohri et al 2001). 
Table 1. Summary of the properties and dimensions of the two segmented scintillator prototypes.

\begin{tabular}{lllllll}
\hline $\begin{array}{l}\text { Prototype } \\
\text { segmented } \\
\text { scintillator }\end{array}$ & $\begin{array}{l}\text { Scitillator } \\
\text { density } \\
\left(\mathrm{g} \mathrm{cm}^{-3}\right)\end{array}$ & $\begin{array}{l}\text { Scintillator } \\
\text { thickness } \\
(\mathrm{mm})\end{array}$ & $\begin{array}{l}\text { Pixel } \\
\text { format }\end{array}$ & $\begin{array}{l}\text { Pixel } \\
\text { pitch } \\
(\mathrm{mm})\end{array}$ & $\begin{array}{l}\text { Active } \\
\text { area } \\
\left(\mathrm{mm}^{2}\right)\end{array}$ & $\begin{array}{l}\text { Septal wall } \\
\text { thickness } \\
(\mathrm{mm})\end{array}$ \\
\hline CsI-1 & 4.51 & 11.4 & $120 \times 60$ & 1.016 & $122 \times 61$ & 0.05 \\
BGO-1 & 7.13 & 11.3 & $120 \times 60$ & 1.016 & $122 \times 61$ & 0.05 \\
\hline
\end{tabular}

\section{Methods}

\subsection{Segmented scintillator prototypes}

The two segmented scintillator prototypes employed in this study have been described previously (Wang et al 2009b) and a summary follows. Specifications for these prototypes (manufactured by Saint-Gobain Crystals, OH, USA) are given in table 1. The prototypes consist of $\sim 11.4 \mathrm{~mm}$ thick CsI:Tl and $\sim 11.3 \mathrm{~mm}$ thick BGO detectors, having estimated $\mathrm{x}$-ray detection efficiencies of $\sim 25 \%$ and $39 \%$, respectively (Wang et al 2009a). Each detector consists of $120 \times 60$ scintillator elements arranged in a two-dimensional grid with an elementto-element pitch of $1.016 \mathrm{~mm}$, resulting in an active area of $\sim 122 \times 61 \mathrm{~mm}^{2}$. Each scintillator element comprises a scintillating crystal surrounded by $\sim 0.05 \mathrm{~mm}$ thick septal walls consisting of a polymer reflector and transparent glue. The walls, which act as a light barrier to limit spatial resolution degradation, have a reflectivity of $\sim 90 \%$, resulting in some degree of light sharing between adjacent elements. The choice of the septal wall thickness in these prototypes was motivated by the desire to improve element-to-element alignment in the manufacturing process, as compared to an earlier prototype (Sawant et al 2006). While thinner walls present the added advantage of higher volumetric fill factor (the fraction of detector volume efficiently absorbing radiation and generating light), they may allow more light spread, resulting in poorer spatial resolution performance. Ideally, this performance should only be limited by the spread of the secondary Compton electrons within the scintillator (Sawant et al 2006). Figure 1 shows both detectors resting on a high-contrast surface. Under illumination by white light, while the BGO detector exhibits excellent transparency, the CsI:Tl detector is more opaque as a result of a higher degree of optical self-scattering within the scintillating crystals as well as additional scattering at the rougher surfaces of the crystals. (For the respective emission spectra of these scintillators, peaked at $\sim 480 \mathrm{~nm}$ for BGO and $\sim 565 \mathrm{~nm}$ for CsI:Tl (Saint Gobain Crystals), light transmission might be somewhat different.)

Each segmented scintillator prototype was coupled to an indirect detection AMFPI array operated in conjunction with a custom set of acquisition electronics (Huang et al 1999). (In this paper, the imaging systems incorporating the CsI:Tl and BGO prototypes will be referred to as CsI-1 and BGO-1, respectively). The array design consists of a pixel format of $1024 \times 1024$ and a pitch of $0.127 \mathrm{~mm}$, resulting in an area of $\sim 13 \times 13 \mathrm{~cm}^{2}$. Arrays of this design have been described and characterized in an earlier publication and have an optical fill factor of $\sim 80 \%$ (Antonuk et al 2009). The choice of this high resolution array, which has a pixel pitch eight times smaller than that of the scintillators, was motivated by the desire to achieve rapid and optimal registration of the scintillator elements with array pixels during set up of the apparatus in the treatment room. Using this array, good registration was simply and quickly attained through angular alignment of the grid of septal walls with the gate and data lines of the array-without further need for registration of the scintillator elements (Wang et al 2009b). Such over-sampling allows the acquisition of x-ray images that give a pictorial representation of the pixel structure of the segmented scintillators, from which precise binning 


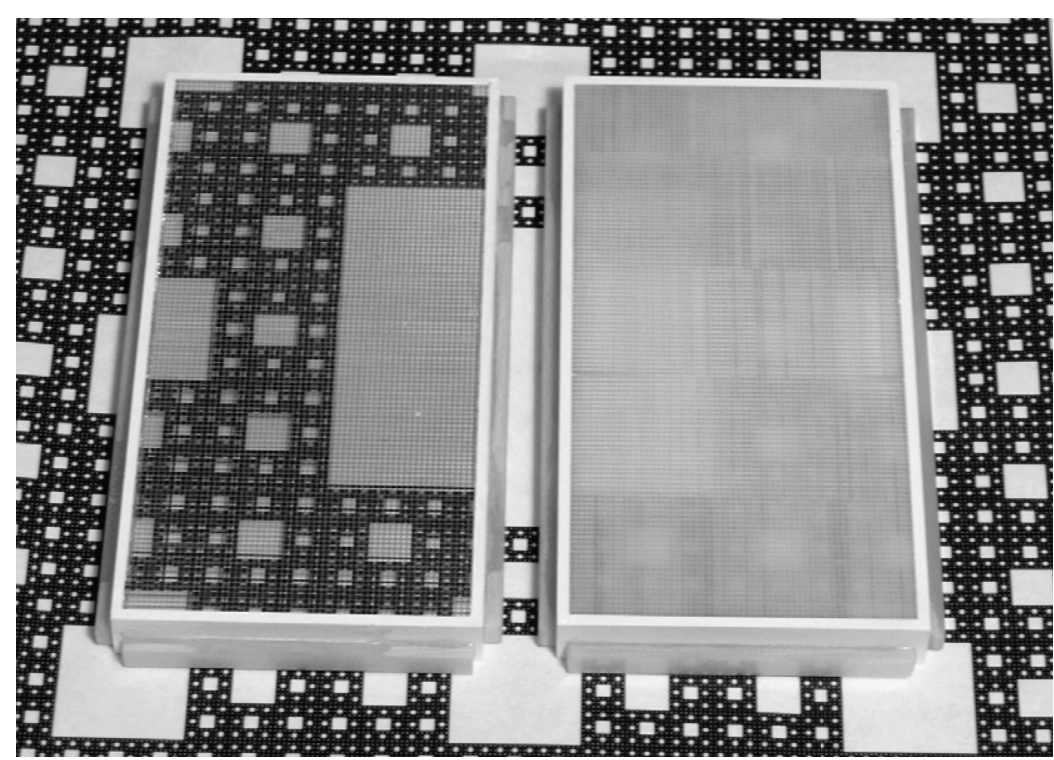

Figure 1. Photograph showing the BGO (left) and CsI:Tl (right) segmented scintillator prototypes resting on a high contrast print depicting a Sierpinski carpet. For each scintillator, a grid of vertical and horizontal lines corresponding to the septal walls is visible in the photo.

to the size of the scintillator elements is achieved. Examples of such images are shown in figure 2 for BGO-1 and CsI-1. Note that for both scintillators, as a result of the scintillator assembly process, the element-to-element alignment is better in the vertical direction. Also, note that while the septal walls for CsI-1 (figure 2(a)) appear darker than the surrounding scintillating crystals, corresponding to lower light output, the relative shading is reversed for BGO-1. This unexpected result for BGO-1, where the septal walls appear to emit more light than the surrounding scintillating crystals can probably be attributed to the large mismatch in the index of refraction between the BGO crystals and the transparent glue (2.15 for BGO and 1.55 for the glue)_leading to the entrapment and channeling of light along the septal walls. For each scintillator, close physical contact with the array was maintained simply by means of the weight of the scintillator. Optical coupling between the scintillator and the array was maintained without the use of an additional coupling medium. For CsI-1, the high optical conversion gain of the scintillating crystals $\left(\sim 54\right.$ photons $\mathrm{keV}^{-1}$ compared to only $\sim 9$ photons $\mathrm{keV}^{-1}$ for BGO crystals $)^{2}$ required the use of an $\sim 0.25 \mathrm{~mm}$ thick neutral density filter with $\sim 21 \%$ light transmission. The filter was positioned between the scintillator and the array to help avoid array pixel saturation. For both prototypes, an $\sim 1 \mathrm{~mm}$ thick Cu plate was positioned directly over the scintillators. The $\mathrm{Cu}$ plate acts as an optical mirror reflector as well as a radiation buildup layer, and also serves to absorb scattered radiation. Finally, for purposes of comparison, measurements were also conducted with a conventional MV AMFPI, consisting of a Lanex Fast-B phosphor screen $\left(133 \mathrm{mg} \mathrm{cm}^{-2} \mathrm{Gd}_{2} \mathrm{O}_{2} \mathrm{~S}\right.$ : Tb, Eastman Kodak, Rochester, NY), an overlying $\sim 1 \mathrm{~mm}$ thick $\mathrm{Cu}$ plate, and the same AMFPI array and associated acquisition electronics used for the prototype scintillators.

2 Saint-Gobain Crystals Product data sheet for scintillation crystal arrays and assemblies, Saint-Gobain Crystals, OH, USA. 


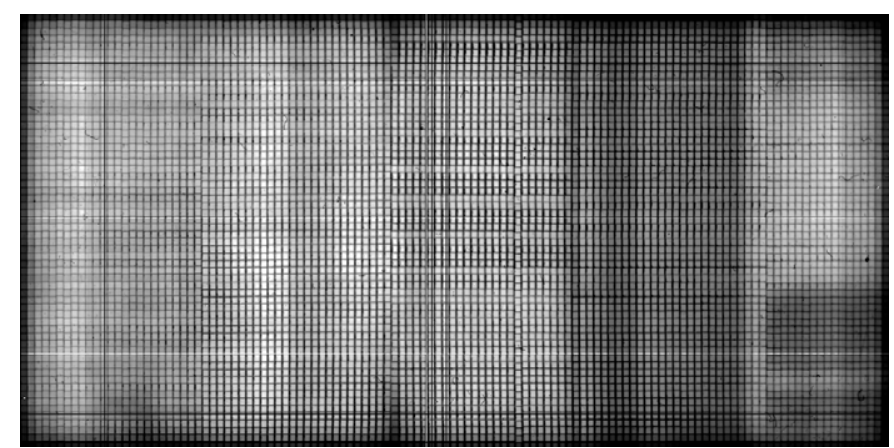

(a)

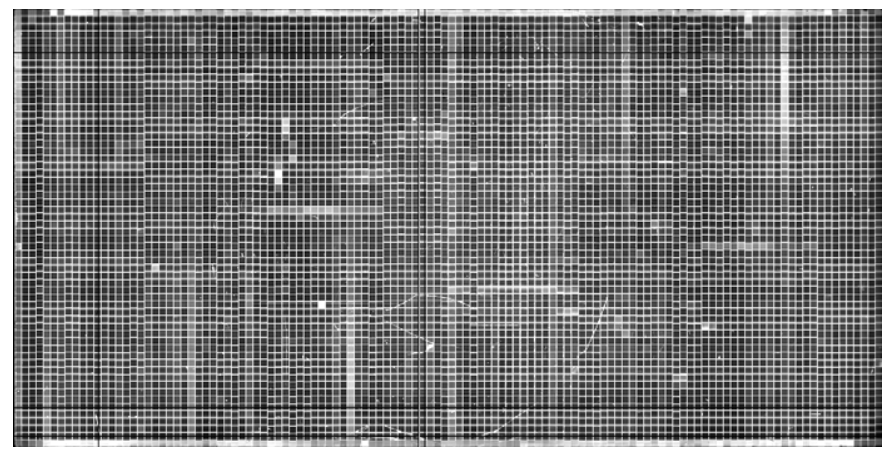

(b)

Figure 2. Flood images acquired at $6 \mathrm{MV}$ at a dose of $\sim 0.133 \mathrm{cGy}$ using (a) CsI-1 and (b) BGO-1. Note that the observed grid of lines with lower signal (darker) for CsI-1 and higher signal (whiter) for BGO-1 correspond to the septal walls. See the text for further details.

\subsection{Experimental technique and apparatus}

In order to explore the potential of the prototype segmented scintillators for low-contrast soft-tissue CBCT imaging, a bench-top scanning system allowing the acquisition of MV tomographic images has been constructed. The system consists of an aluminum frame that supports a solid cylindrical rod made of water-equivalent material (solid water) onto which phantoms can be inserted, as seen in figures $3(a)$ and $(b)$. Each phantom consists of a $4 \mathrm{~cm}$ thick disk with a hole drilled through the center to allow the phantom to slip onto the rod. During image acquisition, the rod is made to rotate around its axis above the scintillator, while keeping the $\mathrm{x}$-ray source at a fixed position. This relatively simple configuration allowed tomographic scans to be obtained by controlling the rotation of the phantom rather than controlling the rotation of the x-ray source and imager. The rotation of the phantom was controlled by a hybrid stepping motor equipped with a 14:1 speed-reducing gearbox operated at a constant speed. The angular velocity of the phantom, as well as the start and stop of the motor, were controlled by a high accuracy stepper motor controller card connected to a host computer via a USB port. Three different phantoms were used to characterize CsI-1, BGO-1 and the conventional MV AMFPI. One phantom, referred to as the contrast phantom, has three holes into which tissue-equivalent objects can be inserted. Details of this phantom are shown in figure 4. A total of 12 different, $\sim 7 \mathrm{~cm}$ long, cylindrical objects of various densities were used. Designations and relative electron densities of these objects are summarized in table 2. A second phantom made with the same material and dimensions (referred to as the uniform 

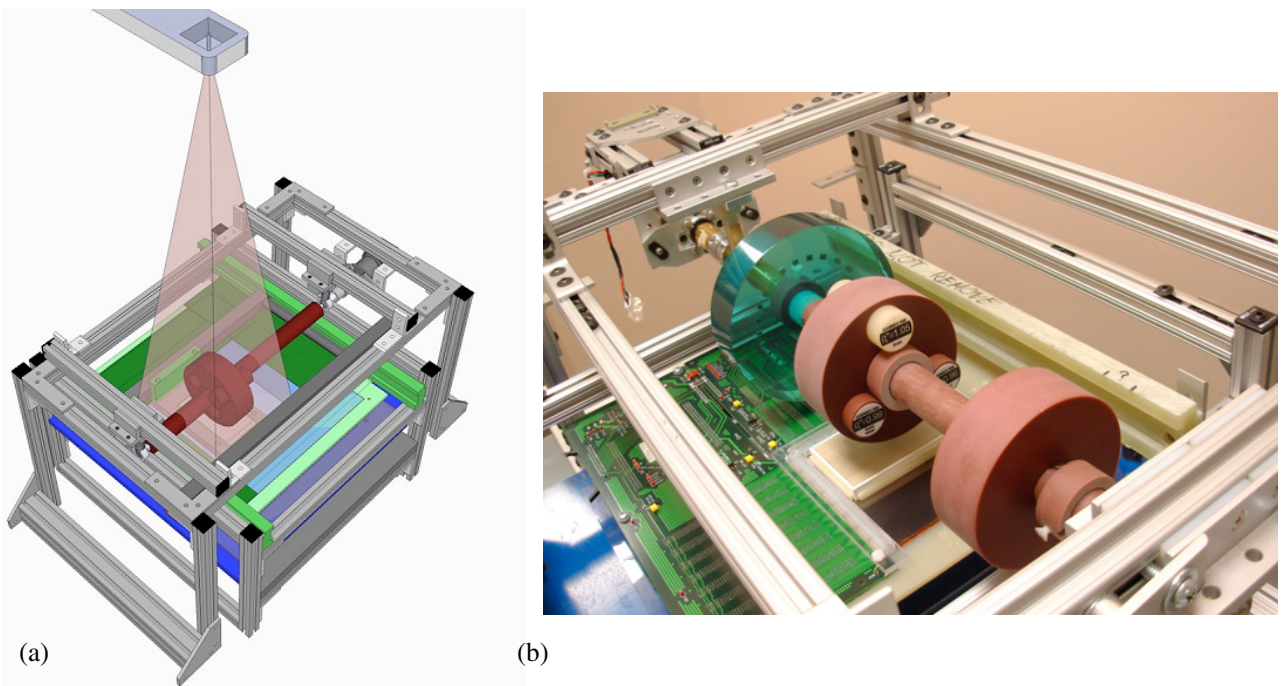

(b)

Figure 3. (a) Conceptual drawing of the cone-beam CT bench-top system used in the study, including the radiation source. (b) Photograph showing a close up of the system clearly depicting the three phantoms mounted above the CsI:Tl segmented scintillator prototype and the underlying AMFPI array.

(This figure is in colour only in the electronic version)

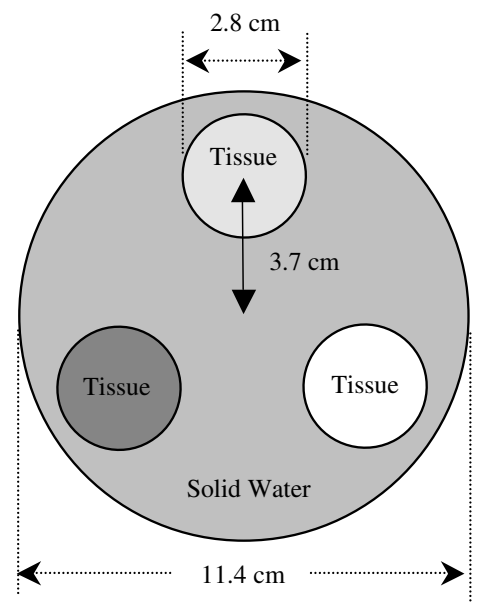

Figure 4. Cross-sectional drawing of the contrast phantom with various dimensions indicated. The phantom is made of solid water into which cylindrical holes are drilled in order to accommodate three tissue-equivalent objects at a time.

phantom) has no inserted objects or holes and was used to provide a reference for the cupping artifact correction (discussed below). Finally, a third phantom, referred to as the resolution phantom, was used to characterize the spatial resolution performance of the various MV AMFPI configurations (CsI-1, BGO-1 and conventional). This phantom consists of an epoxy mix and contains $2 \mathrm{~mm}$ thick aluminum contrast line-pair inserts with resolution sections ranging from 1 to $21 \mathrm{lp} \mathrm{cm}^{-1}$, in steps of $1 \mathrm{lp} \mathrm{cm}^{-1}$ (High Resolution Module, CTP528, The Phantom Laboratory, Salem, NY). 
Table 2. List of designations, densities and electron densities relative to water for the tissueequivalent objects examined in this study (Tissue Characterization Phantom, Gammex 467, Gammex rmi, Middleton, WI). Note that there are two entries for solid water material: one for the material of the main body of the contrast phantom and the other for the tissue-equivalent object inserted in that body. The inclusion of this solid water object in the study was motivated by the fact that it provided the lowest electron density difference relative to the solid water of the main body $(2.76 \%)$ - offering a challenging test for visualization of low contrast objects at the lowest available dose. The last column in the table gives the percentage difference in electron density between the tissue-equivalent objects and the solid water of the main body of the contrast phantom.

\begin{tabular}{llll}
\hline $\begin{array}{l}\text { Designation of } \\
\text { tissue-equivalent } \\
\text { object }\end{array}$ & $\begin{array}{l}\text { Physical } \\
\text { density } \\
\left(\mathrm{g} \mathrm{cm}^{-3}\right)\end{array}$ & $\begin{array}{l}\text { Electron } \\
\text { density relative } \\
\text { to water }\end{array}$ & $\begin{array}{l}\text { Electron } \\
\text { density } \\
\text { difference (\%) }\end{array}$ \\
\hline Lung (LN-300) & 0.29 & 0.280 & 72.44 \\
Lung (LN-450) & 0.44 & 0.429 & 57.78 \\
Adipose (AP6) & 0.94 & 0.925 & 8.96 \\
Breast & 0.98 & 0.954 & 6.10 \\
Solid water (object) & 1.017 & 0.988 & 2.76 \\
Solid water (phantom) & 1.046 & 1.016 & 0.00 \\
Brain & 1.053 & 1.049 & 3.25 \\
Liver (LV1) & 1.097 & 1.065 & 4.82 \\
Inner Bone & 1.143 & 1.096 & 7.87 \\
Bone (B200) & 1.154 & 1.106 & 8.86 \\
Bone (CB2-30\% mineral) & 1.335 & 1.280 & 25.98 \\
Bone (CB2-50\% mineral) & 1.56 & 1.470 & 44.69 \\
SB3 cortical bone & 1.825 & 1.697 & 67.03 \\
\hline
\end{tabular}

MV CBCT images were acquired using a $6 \mathrm{MV}$ photon beam from a Varian radiotherapy linear accelerator (LINAC) operated at a dose rate of 100 Monitor Units (MU) $\mathrm{min}^{-1}$. The LINAC was calibrated so that $1 \mathrm{MU}$ delivers a dose of $0.8 \mathrm{cGy}$ for a $10 \times 10 \mathrm{~cm}^{2}$ field at $10 \mathrm{~cm}$ water depth and at a source-to-detector distance (SDD) of $100 \mathrm{~cm}$. At this dose rate, the LINAC delivers $\sim 36$ beam pulses per MU, corresponding to a dose of $\sim 0.022 \mathrm{cGy}$ per pulse. In this paper, the dose for a tomographic scan is reported in terms of the LINAC's radiation output in cGy, assuming the aforementioned correspondence between dose in cGy and the delivered MUs under calibration conditions. Beam pulses, which are typically $5 \mu \mathrm{s}$ long, were generated at a frequency of $60 \mathrm{~Hz}$, corresponding to a time interval between pulses of $\sim 16.7 \mathrm{~ms}$. These pulses were used to trigger array readout, allowing synchronization between radiation delivery and image acquisition. Given the desire to acquire projection images at the lowest dose available, corresponding to a single beam pulse, each image must be read out within the $16.7 \mathrm{~ms}$ time interval between consecutive beam pulses. However, due to limitations of the electronic acquisition system (Huang et al 1999) which allows a minimum readout time of $\sim 113 \mathrm{~ms}$ for addressing the 1024 gate lines of the AMFPI array, it was necessary to use a combination of simultaneous readout of multiple gate lines and partial array readout to accomplish this objective. (The readout speed of the acquisition system is limited by the conversion rate of the 18-bit analog-to-digital converters as well as the 16:1 multiplexing of the data, resulting in a minimum readout time of $\sim 110 \mu \mathrm{s}$ for a single gate line.) As a result, only a total of 400 gate lines were addressed with each four consecutive lines being addressed simultaneously, resulting in a total of 100 binned lines and a readout time of $\sim 14 \mathrm{~ms}$. Such a configuration for array readout achieves pixel binning, which partially fulfills the intended matching of the scintillator elements, without causing any adverse affects 
on imager performance. The corresponding active area of the array was $\sim 5.1 \times 13 \mathrm{~cm}^{2}$-an area sufficiently large to accommodate the projected area of the contrast phantom $(\sim 4.2 \times$ $12.0 \mathrm{~cm}^{2}$ ). For all AMFPI configurations, the SDD was set to $\sim 130 \mathrm{~cm}$ and the field size to $\sim 9 \times 13 \mathrm{~cm}^{2}$ at isocenter.

\subsection{Image acquisition and analysis}

For each tomographic acquisition, a total of 8000 projection images were acquired corresponding to a total of 44 complete $360^{\circ}$ scans, with each scan comprising 180 images. The total dose delivered per scan was $\sim 4$ cGy. To investigate performance at higher doses, images from different scans were combined resulting in averaged scans with equivalent doses corresponding to a multiple of $4 \mathrm{cGy}$. In this case, the large number of scans acquired allowed the study of dose dependence as well as the determination of the statistical error in the measurements (obtained through calculation of the standard deviation derived from the data of the multiple scans). For the case of BGO-1, a total of 2000 MUs were delivered prior to the start of the measurements in order to attain a stable scintillator light output that is largely independent of accumulated dose. This pre-irradiation was necessary since the BGO-1 signal response is known to exhibit an initial sharp decline of $\sim 17 \%$ with increasing cumulative dose before reaching an asymptotic level (Wang et al 2009b). For each MV AMFPI configuration (BGO-1, CsI-1 or conventional) a set of 100 flood images were also obtained in the absence of the phantoms along with a further 100 dark images to provide gain and offset correction parameters for processing of the projection images of the tomographic scans. A Feldkampbased algorithm employing a ramp filter was used to reconstruct the spatial distribution of attenuation coefficients for the phantoms using gain and offset-corrected projection and flood images (Wang et al 2008). Prior to image reconstruction, all projection images were filtered by means of a $3 \times 3$ median filter in order to remove array pixel and line defects. In addition, the images were binned in an $8 \times 2$ format (gate $\times$ data line direction) so as to match the elementto-element pitch of the scintillators $\left(1.016 \times 1.016 \mathrm{~mm}^{2}\right)$. (Note that, in the data line direction, only $\times 2$ binning is required since array pixels have already been binned in a $1 \times 4$ format by means of multiple gate line readout.) The reconstructed voxel and slice thickness were chosen to be $1.016 \mathrm{~mm}$, matching the element-to-element pitch of the scintillators. Unless otherwise stated, all results shown correspond to the sum of five consecutive slices, resulting in a slice thickness of $\sim 5 \mathrm{~mm}$. All reconstructed images, except for those of the resolution phantom, were subjected to a cupping artifact correction to remove a background trend as well as ring artifacts. While the background trend, which manifests itself as a general increase in signal along the radial direction of the phantom, is due to beam hardening, ring artifacts are likely due to non-uniform detector response. Figure 5 shows an example of a reconstructed image of the contrast phantom before and after the application of the cupping correction. The correction consists of mapping the average signal as a function of radial distance in the background region, excluding the regions of the inserts (Wang et al 2008). In order to compare results from different sets of inserts as well as different AMFPI configurations, the signal in the background was normalized to values obtained from corresponding reconstructed images of the uniform phantom. After application of the cupping correction, the reconstructed images exhibit a more uniform response, as seen in figure 5(b).

\subsection{Performance evaluation}

Performance of various MV AMFPI configurations was characterized in terms of contrast (Contrast), noise (Noise) and contrast-to-noise-ratio $(C N R)$ of the tissue-equivalent objects 


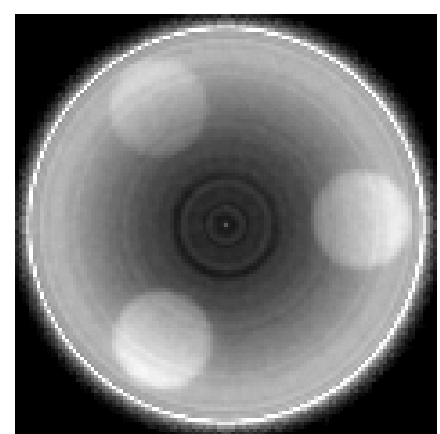

(a)

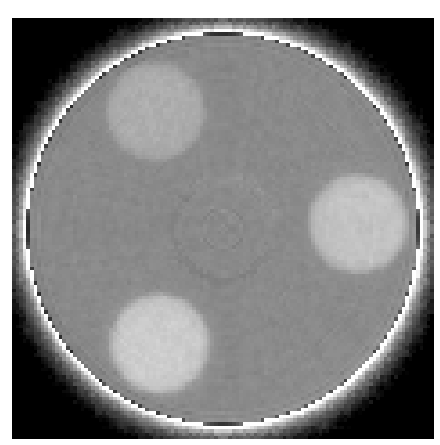

(b)

Figure 5. Reconstructed images of the contrast phantom embedded with three tissue-equivalent objects (a) before and (b) after the application of a cupping artifact correction. The images were obtained with BGO-1 at a scan dose of 16 cGy. Note the presence of a ring-like artifact close to the center of the phantom that was not removed by the correction. This artifact is likely due to the presence of unintended air gaps between the phantom and the solid water rod that supports it.

relative to the surrounding water-equivalent background in the reconstructed images of the contrast phantom. The contrast of a given object was calculated (in Hounsfield units, HU) as follows:

$$
\text { Contrast }=\frac{S_{\mathrm{obj}}-S_{\mathrm{water}}}{S_{\mathrm{water}}} \times 1000(\mathrm{HU})
$$

where $S_{\text {obj }}$ and $S_{\text {water }}$ represent the mean signal in the object and solid water regions, obtained through averaging signals from 185 and 555 voxels, respectively. The choice of these regions of interest excluded the edge of the objects and the phantom as well as the center of the phantom.

Similarly, the noise in the object was calculated from

$$
\text { Noise }=\frac{\sigma_{\text {obj }}}{S_{\text {water }}} \times 1000(\mathrm{HU})
$$

where $\sigma_{\mathrm{obj}}$ represents the standard deviation of the signal in the object. Therefore, the CNR was calculated from

$$
\mathrm{CNR}=\frac{S_{\mathrm{obj}}-S_{\mathrm{water}}}{\sigma_{\mathrm{obj}}}
$$

\section{Results}

\subsection{Reconstructed images}

Examples of reconstructed images of the contrast phantom obtained with BGO-1 for a slice thickness of $\sim 5 \mathrm{~mm}$ are shown in figure 6 . The images, depicting all 12 tissue-equivalent objects used, were obtained at a scan dose of $\sim 4$ cGy corresponding to a single beam pulse per projection image. These and similar images of the contrast phantom were used to derive the following results for Contrast, Noise and CNR. Reconstructed images of the contrast phantom including the three objects that exhibit the lowest relative electron density difference relative to the solid water background are shown in figure 7 for various AMFPI configurations. At a total scan dose of $\sim 4$ cGy, the images obtained with BGO-1 and CsI-1 (figures 7(a) 


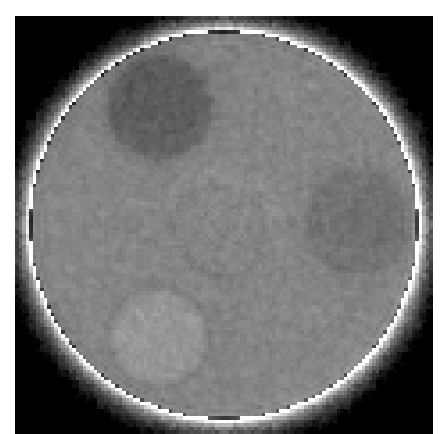

(a)

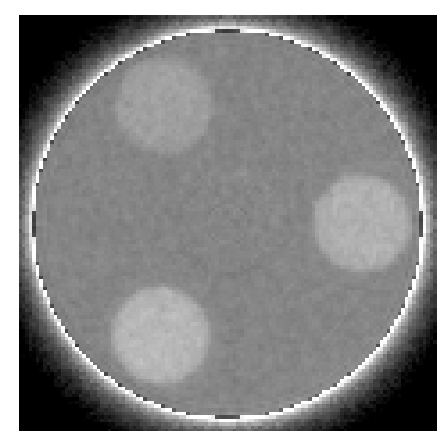

(c)

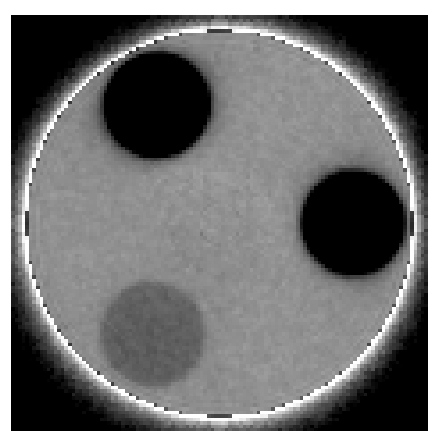

(b)

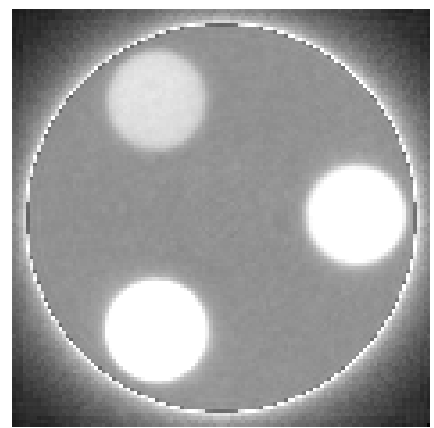

(d)

Figure 6. Reconstructed images of the contrast phantom embedded with various tissue-equivalent objects. The objects shown correspond to relative electron densities of (clockwise from the top): (a) $0.954,0.988,1.049$; (b) $0.280,0.429,0.925$; (c) 1.065, 1.096, 1.106; and (d) 1.280, 1.470, 1.697. The images were obtained with BGO-1 at a scan dose of $\sim 4 \mathrm{cGy}$.

and (b)) exhibit significantly higher contrast resolution compared to that of the conventional MV AMFPI (figure 7(c)). Such good performance is the result of the more efficient use of the incident $\mathrm{x}$-rays by BGO-1 and CsI- 1 achieved by virtue of $\mathrm{DQE}(0)$ values of $\sim 20 \%$, and $\sim 12 \%$, respectively (Wang et al 2009b) — compared to only $\sim 1 \%$ for the conventional MV AMFPI. While all the tissue-equivalent objects are clearly seen for BGO-1 and CsI-1, including the water-equivalent object (see table 1), none of these objects are easily discerned for the conventional MV AMFPI. However, with the use of 40 times more dose (160 cGy) for the conventional MV AMFPI, the objects become visible with slightly improved edge definition compared to the BGO-1 and CsI-1 images-a consequence of the superior spatial resolution performance of the phosphor screen. Finally, BGO-1 exhibits slightly better spatial resolution performance compared to CsI-1, confirming the findings from a previous study of the prototypes involving measurements of the modulation transfer function (Wang et al 2009 b). These observations on spatial resolution performance are consistent with the results of reconstructed images obtained with the resolution phantom, shown in figure 8 . These images depict some of the line-pair inserts of the resolution phantom with sizes ranging from 1 to $11 \mathrm{lp} \mathrm{cm}^{-1}$. While for BGO-1 (figure 8(a)) it is possible to discern the inserts representing a resolution of $4 \mathrm{lp} \mathrm{cm}^{-1}$ (corresponding to a line spacing of $\sim 1.25 \mathrm{~mm}$ ), for CsI-1 (figure 8(b)) the highest discernable insert is that at $3 \mathrm{lp} \mathrm{cm}^{-1}$ (corresponding to a line spacing of $\sim 1.66 \mathrm{~mm}$ ). By comparison, the image obtained with the conventional MV AMFPI (figure 8(c)) demonstrates sharper edge definition and a clear definition of the inserts up to that at $4 \mathrm{lp} \mathrm{cm}^{-1}$. 


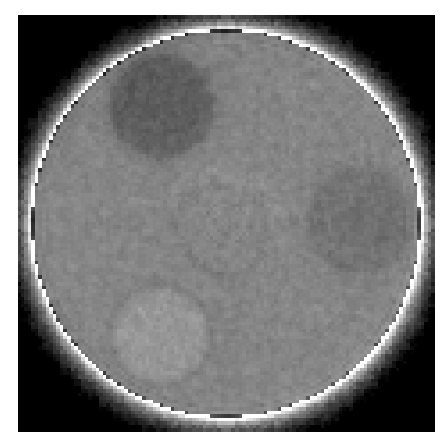

(a)

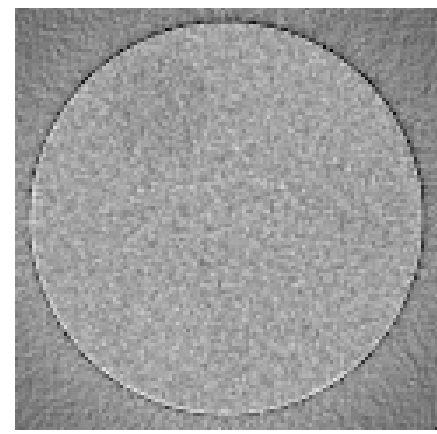

(c)

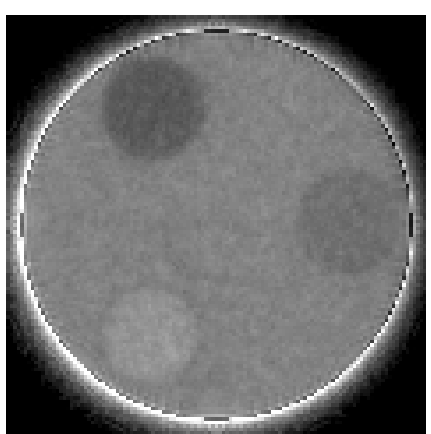

(b)

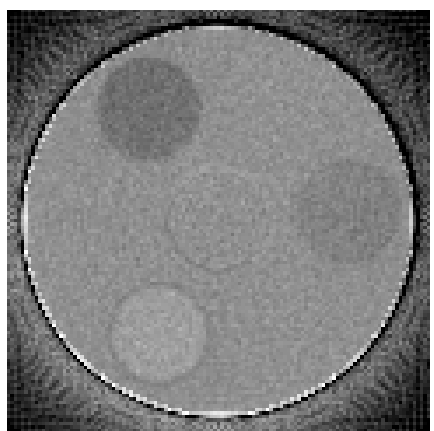

(d)

Figure 7. Reconstructed images of the contrast phantom embedded with three tissue-equivalent objects corresponding to relative electron densities of (clockwise from the top): $0.954,0.988$ and 1.049. The images were obtained at a scan dose of $\sim 4 \mathrm{cGy}$ with (a) BGO-1, (b) CsI-1 and (c) the conventional MV AMFPI. The image in (d) was obtained with the conventional MV AMFPI at 160 cGy by averaging 40 tomographic scans.

\subsection{Contrast, noise and CNR}

Figures 9(a) and (b) show Contrast and Noise results as a function of dose for the breast tissue-equivalent object obtained using BGO-1 and CsI-1 as well as with the conventional MV AMFPI. Within statistical error, Contrast exhibits no clear dependence on dose or on the type of detector used-a consequence of the fact that the value of this metric is largely determined by the properties of the object imaged. Noise, shown in figure 9(b), decreases as a function of dose as a result of the increase in the x-ray quanta sampled. For a given dose, while CsI-1 is expected to exhibit higher Noise values compared to BGO-1 by virtue of its inferior x-ray detection efficiency ( $\sim 25 \%$ compared to $\sim 39 \%)$, the more pronounced light spreading for CsI-1, which causes inferior spatial resolution performance, leads to reduced voxel-to-voxel signal variations in the reconstructed images-resulting in slightly lower values of Noise. As expected, the conventional MV AMFPI exhibits the highest Noise values due to the substantially lower number of $x$-ray quanta sampled ( $\sim 2 \%$ x-ray detection efficiency).

Figure 10(a) shows values for $C N R^{2}$ derived from the Contrast and Noise results in figure 9. For all detector configurations, $C N R^{2}$ is observed to increase with increasing dose, mainly due to the corresponding decrease of Noise. This dependence follows a linear trendas demonstrated by the lines running through the data points which correspond to linear fits of the data, plotted on a $\log$ scale. Figure 10(b) illustrates $C N R^{2}$ performance as a function of 


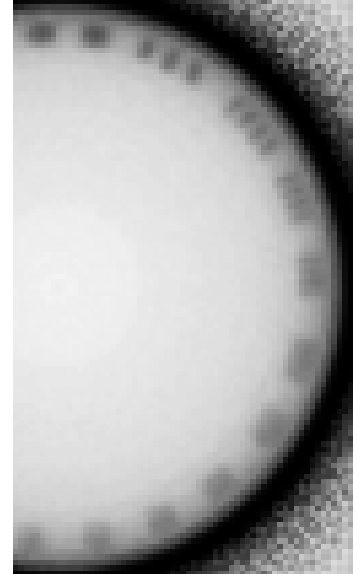

(a)

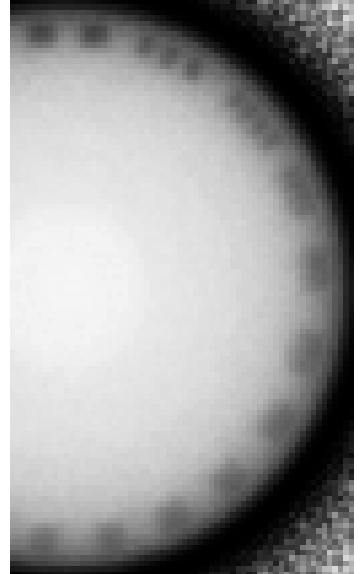

(b)

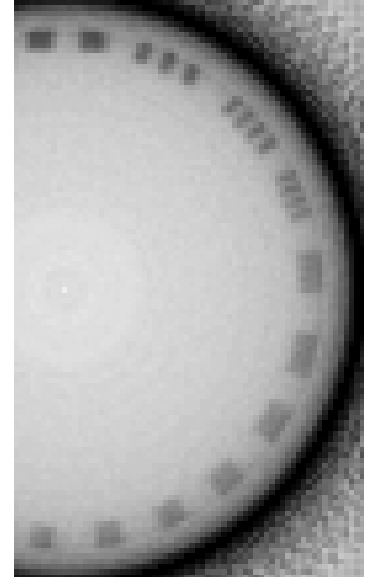

(c)

Figure 8. Reconstructed images of the resolution phantom obtained with (a) BGO-1, (b) CsI-1 and (c) the conventional MV AMFPI at a scan dose of 16,16 and $160 \mathrm{cGy}$, respectively. The images were obtained using a slice thickness of $1.016 \mathrm{~mm}$, corresponding to the element-to-element pitch of the BGO-1 and CsI-1 segmented scintillators. Note that the choice of doses used to produce these images was guided by the desire to produce good images whose quality is not limited by image noise. Also, note that for these images the gray scale has been inverted in order to enhance presentation. The line-pair inserts shown in the images represent spatial resolutions of (clockwise from the top) $1,2,3,4,5,6,7,8,9,10$ and $11 \mathrm{lp} \mathrm{cm}^{-1}$ corresponding to a line spacing distance of $\sim 5.00,2.50,1.66,1.25,1.00,0.83,0.71,0.62,0.55,0.50$ and $0.45 \mathrm{~mm}$, respectively.

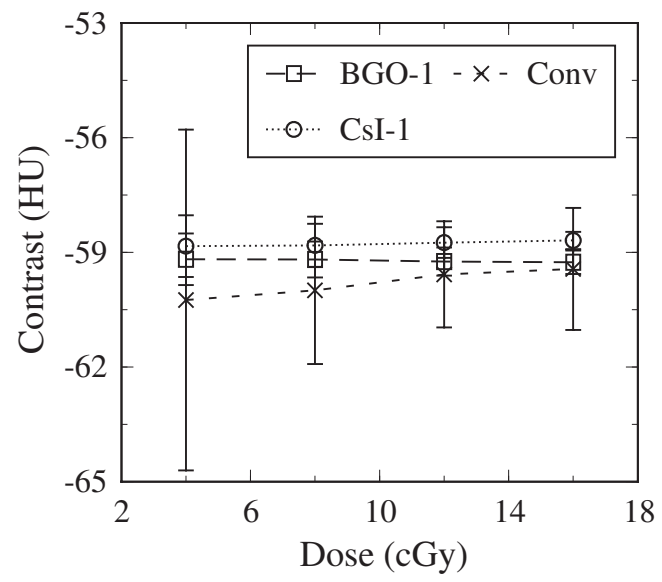

(a)

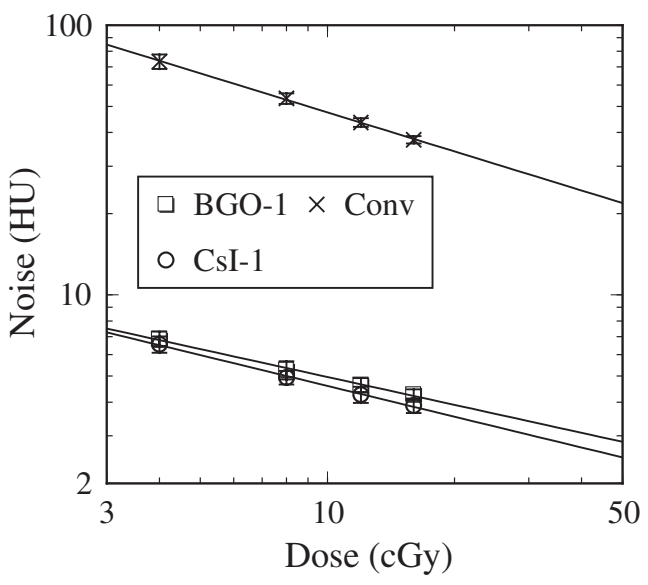

(b)

Figure 9. (a) Contrast and (b) Noise for the breast tissue-equivalent object as a function of total scan dose. Results are shown for BGO-1 and CsI-1, as well as for the conventional MV AMFPI. The lines connecting the data points in (a) are drawn to guide the eye, while the lines in (b) are fits to the data. Statistical error bars are illustrated in this and the following figures, except as noted.

slice thickness up to $\sim 10 \mathrm{~mm}$ for BGO- 1 and CsI- 1 at a scan dose of $\sim 4 \mathrm{cGy}$. These results also exhibit a linear increase with increasing slice thickness. The linear behavior observed in figures 10(a) and (b) for the breast object, and similarly observed for all objects studied, is as expected and is due to the inverse proportionality between the square of Noise (Noise ${ }^{2}$ ) and 


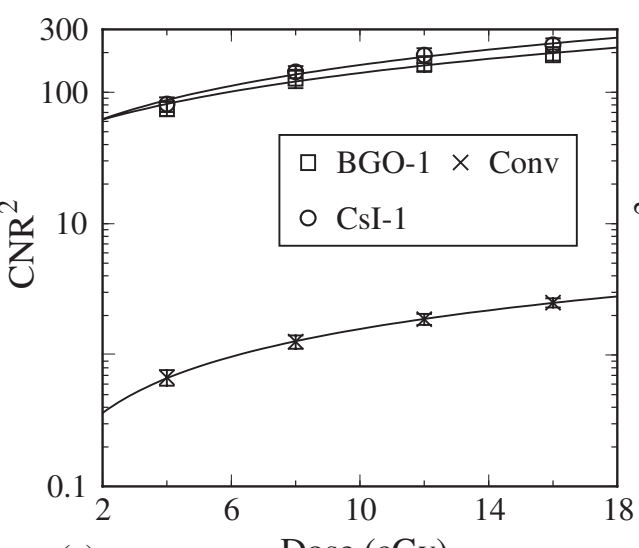

(a)

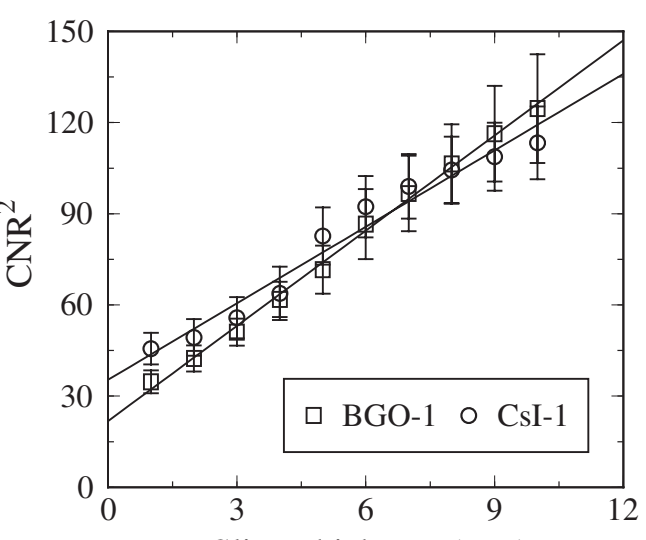

(b) Slice Thickness (mm)

Figure 10. $C N R^{2}$ as a function of (a) total scan dose and (b) slice thickness. Results for the breast tissue-equivalent object are shown for BGO-1 and CsI-1, as well as for the conventional MV AMFPI. Results in (a) are plotted on a log scale to enhance clarity of presentation for the data corresponding to the conventional MV AMFPI. The lines connecting the points in (a) and (b) are linear fits to the data.

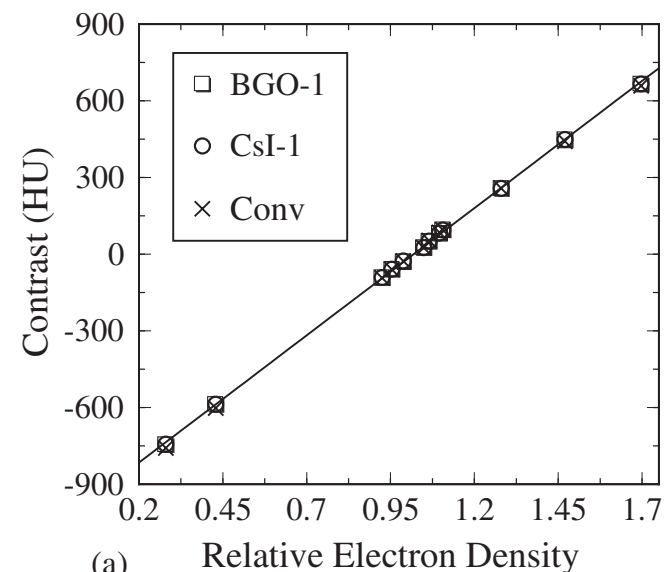

(a) Relative Electron Density

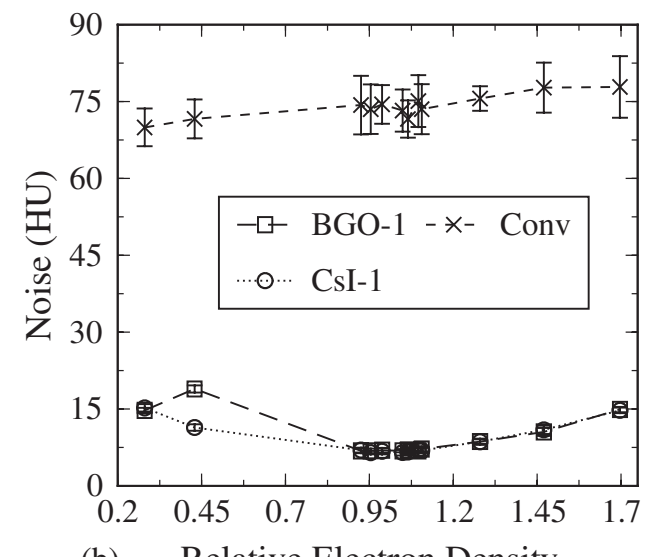

(b) Relative Electron Density

Figure 11. (a) Contrast and (b) Noise as a function of relative electron density for the various tissue-equivalent objects. Results are shown for BGO-1 and CsI-1, as well as for the conventional MV AMFPI, at a total scan dose of $\sim 4 \mathrm{cGy}$. The line appearing in (a) represents a linear fit to the BGO-1 data, while the lines in (b) are drawn to guide the eye. Note that the error bars in (a) have been purposely omitted due to their small size.

the number of sampled quanta (i.e. Noise $e^{2} \propto 1 / N_{\text {tot }}$ where $N_{\text {tot }}$ is the total number of x-ray photons interacting in a given voxel).

Figures 11(a) and (b) show Contrast and Noise, respectively, plotted as a function of the relative electron density of the various tissue-equivalent objects, obtained with BGO-1 and CsI-1 as well as with the conventional MV AMFPI. Contrast is seen to exhibit a fairly linear dependence on relative electron density due to the dominance of Compton interactions at radiotherapy $\mathrm{x}$-ray energies. Specifically, this behavior is the consequence of the linear dependence of the probability of Compton interactions on the electron density of the interaction 


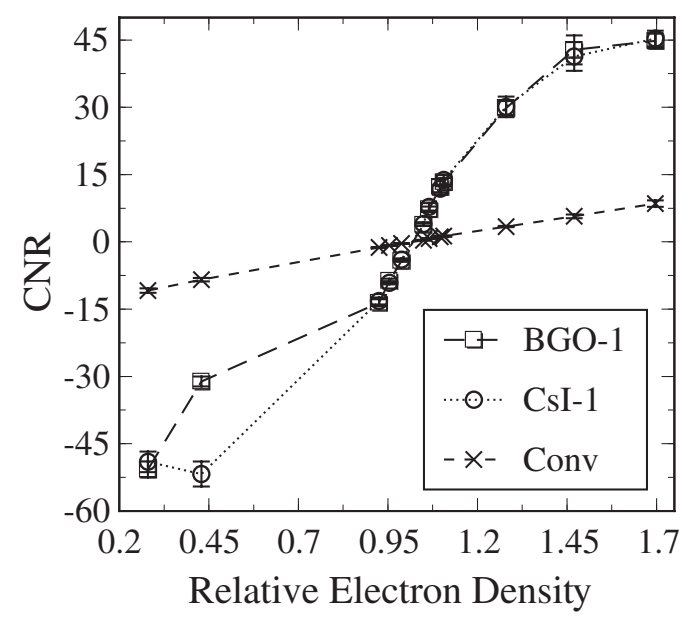

Figure 12. $C N R$ as a function of the relative electron density of the various tissue-equivalent objects. The results were obtained with BGO-1 and CsI-1, as well as with the conventional MV AMFPI, at a total scan dose of $\sim 4 \mathrm{cGy}$. Note that the lines connecting the data points are drawn to guide the eye.

material. The observed values of Contrast indicate that this quantity is a property of the object imaged and is largely independent of the detector configuration. For the two prototypes, Noise values are observed to be similar except for the anomalously high (and repeatable) value for BGO-1 at an electron density of 0.429 . For objects with relative electron densities closer to that of water, Noise is seen to be fairly independent of this quantity. Moreover, for objects with densities much higher or lower than that of water, Noise values are higher. Since such a pattern of behavior is not observed in the results obtained from the conventional MV AMFPI, it is suspected that this pattern is due to the cupping correction. In the case of the MV AMFPI, any uncertainties introduced by the cupping correction are dwarfed by the inherently large values of Noise in the reconstructed images.

Figure 12 shows results for CNR derived from the Contrast and Noise data in figure 11 and plotted as a function of relative electron density. While $C N R$ for the conventional MV AMFPI is seen to be linear for all objects, for BGO-1 and CsI-1 a reasonable degree of linearity is observed only for objects with relative electron densities ranging from $\sim 0.92$ to $\sim 1.11$-due to the corresponding linear behavior of Contrast and constancy of Noise for these objects. The $C N R$ values for objects with densities outside this range exhibit a nonlinear dependence on this metric due to the higher than expected Noise values discussed above. Finally, the observed superior performance of the prototypes compared with that of the conventional MV AMFPI is the result of significantly lower Noise values.

\section{Summary and discussion}

Active matrix flat-panel imagers, currently regarded as the gold standard for portal imaging, utilize a very inefficient $\mathrm{X}$-ray converter in the form of a relatively thick $\mathrm{Gd}_{2} \mathrm{O}_{2} \mathrm{~S}$ : $\mathrm{Tb}$ phosphor screen coupled to a $\sim 1 \mathrm{~mm}$ thick metal plate, resulting in an x-ray detection efficiency of only $\sim 2 \%$ at a photon beam energy of $6 \mathrm{MV}$. However, the incorporation of thick segmented scintillators, as a replacement for such granular phosphor screens, provides a substantial increase in detection efficiency and has shown considerable promise for improving the imaging performance without seriously degrading spatial resolution or contributing additional Swank 
noise due to variations in the generation of optical photons. Toward the realization of practical AMFPIs capable of performance largely limited by the radiation transport within the $\mathrm{x}$-ray converter material, and offering minimal degradation due to optical transport, a number of prototype AMFPIs based on thick BGO and CsI:Tl scintillators have been developed and evaluated by our group for portal (i.e. 2D) imaging. Given the high detection efficiency of these prototypes, combined with the desire to obtain 3D image information in the treatment room for image-guided radiation therapy, it is of interest to investigate these prototypes in the context of low-dose MV CBCT. In this paper, preliminary MV CBCT results from two prototypes based on $\sim 11 \mathrm{~mm}$ thick BGO and CsI:Tl scintillators for MV CBCT are reported and these results are compared to those from a conventional MV AMFPI. The study was performed for 180 projection, $360^{\circ}$ tomographic scans employing the lowest available dose of $\sim 4 \mathrm{cGy}$ (corresponding to one beam pulse per projection). This dose is similar to that typically employed for a patient setup using $\mathrm{kV}$ CBCT or acquiring a single portal image from a conventional MV AMFPI. By virtue of their greatly improved detection efficiency, the prototypes provided reconstructed images allowing visualization of low contrast objects for a $5 \mathrm{~mm}$ slice thickness. For example, an object with an electron density difference of $\sim 2.76 \%$, corresponding to a $C N R$ value of $\sim 4$, was clearly visible in the reconstructed images of the two prototypes. By comparison, the conventional MV AMFPI demonstrated a corresponding $C N R$ value more than ten times smaller, and did not permit delineation of the object. For this imager, much higher doses were required to reduce image noise so as to allow visualization of the object. The level of improved contrast resolution offered by the current prototypes should facilitate soft-tissue visualization in image-guided radiotherapy at practical MV patient doses, and is also expected to benefit portal imaging through improved image quality at current levels of dose or equivalent image quality at substantially lower dose.

While both types of scintillators (BGO and CsI:Tl) have shown definite promise for improving the imaging performance of current MV AMFPIs, BGO provides a number of advantages. For a given thickness, BGO offers higher DQE and superior spatial resolution by virtue of its higher material density and refractive index, respectively. These properties engender opposing effects upon noise, resulting in a $C N R$ performance that is largely equivalent for the two prototypes. However, if ideal optical isolation between scintillator elements could be achieved, the BGO-1 prototype would provide lower noise and thus higher CNR (Wang et al 2008). Furthermore, BGO is non-hygroscopic and provides better material hardness, resulting in easier machining and polishing. Finally, although BGO has a significantly lower light output compared to CsI:Tl, this has minimal influence on performance, given that for BGO-1 the system is input-quantum limited, even at the lowest dose of 1 beam pulse (Wang et al 2009b). However, the present BGO scintillator exhibits an undesirable radiation-induced effect, which consists of a sharp decline of light output within the first few hundred cGy of radiation, requiring the use of a pre-irradiation procedure to stabilize signal (Wang et al 2009b). While previous examinations of the effect of radiation on the performance of BGO crystals have indicated widely varying results (Wei et al 1990, Zhu et al 1991, 1995, Zhu 1998, Georgii et al 1998, Peng et al 2000, Sahu et al 1997), the introduction of $\mathrm{Eu}^{3+}$ as a doping agent may significantly reduce the effect (Wei et al 1990, Zhu et al 1991, 1995, Zhu 1998), facilitating practical implementation of such detectors in a clinical setting.

With a scintillator thickness of $\sim 11 \mathrm{~mm}$, the BGO prototype offers a zero-frequency DQE of $\sim 20 \%-20$ times higher than that of conventional MV AMFPIs. While prototypes with thicker scintillators could further improve the DQE and allow even better soft-tissue visualization at low dose, degradation of spatial resolution due to obliquely incident radiation could mitigate the improvement expected from higher x-ray detection efficiencies (Wang et al 2010). For example, for a large area (e.g., $40 \times 40 \mathrm{~cm}^{2}$ ) clinical imager based on segmented 
scintillators with thicknesses much greater than $\sim 10 \mathrm{~mm}$, x-rays incident at locations away from the central axis will impinge the scintillators at increasingly oblique angles-resulting in a substantial lateral displacement of energy deposition across the scintillator and causing severe degradation of spatial resolution and DQE performance. Future substantial increases in DQE beyond the levels obtained with the present prototypes could potentially be achieved through the use of thicker (i.e. greater than $\sim 10 \mathrm{~mm}$ ) scintillators arranged in a two-dimensionally focused geometry (Wang et al 2010) or, alternatively, through the use of higher density scintillators with thicknesses up to $\sim 10 \mathrm{~mm}$. The strong motivation to improve DQE for MV imagers will doubtless inspire interesting innovations in the coming years.

\section{Acknowledgments}

The authors would like to thank Mike Yeakey, Alan Young, Charles Martelli and Dr Yi Wang for assistance with the establishment of the bench-top CT system. The authors would also like to thank Dr Jeffrey Fessler for providing the cone-beam reconstruction algorithm, and Martin Koniczek with assistance in the utilization of the algorithm. This project is supported by NIH grant R01 CA051397.

\section{References}

Amer A, Marchant T, Sykes J, Czajka J and Moore C 2007 Imaging doses from the Elekta Synergy x-ray cone beam CT system Br. J. Radiol. 80 476-82

Antonuk L E, Zhao Q, El-Mohri Y, Du H, Wang Y, Street R A, Ho J, Weisfield R and Yao W 2009 An investigation of signal performance enhancements achieved through innovative pixel design across several generations of indirect detection, active matrix, flat-panel arrays Med. Phys. 36 3322-39

Barker J L et al 2004 Quantification of volumetric and geometric changes occurring during fractional radiotherapy for head-and-neck cancer using an integrated CT/linear accelerator system Int. J. Radiat. Oncol., Biol., Phys. 59 960-70

Court L, Rosen I, Mohan R and Dong L 2003 Evaluation of mechanical precision and alignment uncertainties for an integrated CT/LINAC system Med. Phys. 30 1198-210

Dawson L A and Jaffray D A 2007 Advances in image-guided radiation therapy J. Clin. Oncol. 25 938-46

El-Mohri Y, Jee K-W, Antonuk L E, Maolinbay M and Zhao Q 2001 Determination of the detective quantum efficiency of a prototype, megavoltage indirect detection, active matrix flat-panel imager Med. Phys. 28 2538-50

Ford E C, Chang J, Mueller K, Sidhu K, Todor D, Mageras G, Yorke E, Ling C C and Amols H 2002 Cone-beam CT with megavoltage beams and an amorphous silicon electronic portal imaging device: potential for verification of radiotherapy of lung cancer Med. Phys. 29 2913-24

Georgii R, Meifll R, Hajdas W, Henschel H, Graf H D, Lichti G G, Neumann-Cosel P v, Richter A and Schonfelder V 1998 Influence of radiation damage on BGO scintillation properties Nucl. Instrum. Methods Phys. Res. A $41350-8$

Ghelmansarai F A, Bani-Hashemi A, Pouliot J, Calderon E, Hernandez P, Mitschke M, Aubin M and Bucci K 2005 Soft tissue visualization using a highly efficient megavoltage cone beam CT imaging system Proc. SPIE 5745 159-70

Groh B A, Siewerdsen J H, Drake D G, Wong J W and Jaffray D A 2002 A performance comparison of flat-panel imager-based MV and kV cone-beam CT Med. Phys. 29 967-75

Guan H, Yin F F and Kim J H 2002 Accuracy of inhomogeneity correction in photon radiotherapy from CT scans with different settings Phys. Med. Biol. 47 N223-31

Huang W, Antonuk L E, Berry J, Maolinbay M, Martelli C, Mody P, Nassif S and Yeakey M 1999 An asynchronous, pipelined, electronic acquisition system for active matrix flat-panel imagers (AMFPIs) Nucl. Instrum. Methods Phys. Res. A 431 273-84

Islam M K, Purdie T G, Norrlinger B D, Alasti H, Moseley D J, Sharpe M B, Siewerdsen J H and Jaffray D A 2006 Patient dose from kilovoltage cone beam computed tomography imaging in radiation therapy Med. Phys. 33 1573-82

Jaffray D A and Siewerdsen J H 2000 Cone-beam computed tomography with a flat-panel imager: initial performance characterization Med. Phys. 27 1311-23

Jaffray D A, Siewerdsen J H, Wong J W and Martinez A A 2002 Flat-panel cone-beam computed tomography for image-guided radiation therapy Int. J. Radiat. Oncol., Biol., Phys. 53 1337-49 
Keller H, Glass M, Hinderer R, Ruchala K, Jeraj R, Olivera G and Mackie T R 2002 Monte Carlo study of a highly efficient gas ionization detector for megavoltage imaging and image-guided radiotherapy Med. Phys. 29 165-75

Langen K M, Meeks S L, Poole D O, Wagner T H, Willoughby T R, Kupelian P A, Ruchala K J, Haimerl J and Olivera G H 2005 The use of megavoltage CT (MVCT) images for dose recomputations Phys. Med. Biol. 50 4259-76

Lewis D G, Swindell W, Morton E J, Evans P M and Xiao Z R 1992 A megavoltage CT scanner for radiotherapy verification Phys. Med. Biol. 37 1985-99

Mackie T R, Balog J, Ruchala K, Shepard D, Aldridge S, Fitchard E, Reckwerdt P, Olivera G, McNutt T and Mehta M 1999 Tomotherapy Semin. Radiat. Oncol. 9 108-17

Meeks S L, Harmon J F, Langen K M, Willoughby T R, Wagner T H and Kupelian P A 2005 Performance characterization of megavoltage computed tomography imaging on a helical tomotherapy unit Med. Phys. 32 2673-81

Monajemi T T, Fallone B G and Rathee S 2006a Thick, segmented $\mathrm{CdWO}_{4}$-photodiode detector for cone beam megavoltage CT: a Monte Carlo study of system design parameters Med. Phys. 33 4567-77

Monajemi T T, Steciw S, Fallone B G and Rathee S 2004 Modeling scintillator-photodiodes as detectors for megavoltage CT Med. Phys. 31 1225-34

Monajemi T T, Tu D, Fallone B G and Rathee S 2006b A bench-top megavoltage fan-beam CT using CdWO $4^{-}$ photodiode detectors: II. Image performance evaluation Med. Phys. 33 1090-100

Morin O, Chen J, Aubin M, Bose S, Gillis A, Bucci M and Pouliot J 2005 Dose calculation using megavoltage cone beam CT imaging Int. J. Radiat. Oncol. Biol. Phys. 63 S62-3

Morin O, Gillis A, Chen J, Aubin M, Bucci M K, Roach M III and Pouliot J 2006 Megavoltage cone-beam CT: system description and clinical applications Med. Dosim. 31 51-61

Mosleh-Shirazi M A, Evans P M, Swindell W, Symonds-Tayler J R N, Webb S and Partridge M 1998a Rapid portal imaging with a high-efficiency, large field-of-view detector Med. Phys. 25 2333-46

Mosleh-Shirazi M A, Swindell W and Evans P M 1998b Optimization of the scintillation detector in a combined 3D megavoltage CT scanner and portal imager Med. Phys. 25 1880-90

Pang G and Rowlands J A 2004 Development of high quantum efficiency, flat panel, thick detectors for megavoltage X-ray imaging: a novel direct-conversion design and its feasibility Med. Phys. 31 3004-16

Peng K C, Lu R S, Ueno K, Wang C H, Wang M Z, Chou F I, Wei Y Y and Hou W S 2000 Low-dose radiation damage and recovery of undoped BGO crystals Nucl. Instrum. Methods Phys. Res. A 452 252-5

Pouliot J et al 2005 Low-dose megavoltage cone-beam CT for radiation therapy Int. J. Radiat. Oncol. Biol. Phys. 61 552-60

Rathee S, Tu D, Monajemi T T, Rickey D W and Fallone B G 2006 A bench-top megavoltage fan-beam CT using $\mathrm{CdWO}_{4}$-photodiode detectors: I. System description and detector characterization Med. Phys. 33 1078-89

Sahu S K, Peng K C, Huang H C, Wang C H, Chang Y H, Hou W S, Ueno K, Chou F I and Wei Y Y 1997 Radiation hardness of undoped BGO crystals Nucl. Instrum. Methods Phys. Res. A 388 144-8

Samant S S and Gopal A 2006 Study of a prototype high quantum efficiency thick scintillation crystal video-electronic portal imaging device Med. Phys. 33 2783-91

Saw C B, Heron D E, Huq M S and Yue N J 2006 Target delineation and localization (IGRT)—part 1 Med. Dosim. 31 1-2

Sawant A et al 2005 Segmented phosphors-MEMS-based high quantum efficiency detectors for megavoltage x-ray imaging Med. Phys. 32 553-65

Sawant A, Antonuk L E, El-Mohri Y, Zhao Q, Wang Y, Li Y, Du H and Perna L 2006 Segmented crystalline scintillators: empirical and theoretical investigation of a high quantum efficiency EPID based on an initial engineering prototype CsI(Tl) detector Med. Phys. 33 1053-66

Seppi E J et al 2003 Megavoltage cone-beam computed tomography using a high-efficiency image receptor Int. J. Radiat. Oncol., Biol., Phys. 55 793-803

Sillanpaa J, Chang J, Mageras G, Yorke E, De Arruda F, Rosenzweig K, Munro P, Seppi E, Pavkovich J and Amols H 2006 Low-dose megavoltage cone-beam computed tomography for lung tumors using a high-efficiency image receptor Med. Phys. 33 3489-97

Wang Y, Antonuk L E, El-Mohri Y and Zhao Q 2009a A Monte Carlo investigation of Swank noise for thick, segmented, crystalline scintillating detectors for radiotherapy imaging Med. Phys. 36 3227-38

Wang Y, Antonuk L E, El-Mohri Y, Zhao Q, Sawant A and Du H 2008 Monte Carlo investigations of megavoltage cone-beam CT using thick, segmented scintillating detectors for soft tissue visualization Med. Phys. 35 145-58

Wang Y, Antonuk L E, Zhao Q, El-Mohri Y and Perna L 2009b High-DQE EPIDs based on thick, segmented BGO and CsI:Tl scintillators: performance evaluation at extremely low dose Med. Phys. 36 5707-18

Wang Y, El-Mohri Y, Antonuk L E and Zhao Q 2010 Monte Carlo investigations of the effect of beam divergence on thick, segmented crystalline scintillators for radiotherapy imaging Phys. Med. Biol. 55 3659-73 
Wei Z Y, Zhu R Y, Newman H and Yin Z W 1990 Radiation resistance and fluorescence of europium doped BGO crystals Nucl. Instrum. Methods Phys. Res. A 297 163-8

Yin F F, Guan H and Lu W 2005 A technique for on-board CT reconstruction using both kilovoltage and megavoltage beam projections for 3D treatment verification Med. Phys. 32 2819-26

Zhu R Y 1998 Radiation damage in scintillating crystals Nucl. Instrum. Methods Phys. Res. A 413 297-311

Zhu R Y, Ma D A and Newman H 1995 Scintillating crystals in a radiation environment Nucl. Phys. B (Proc. Suppl.) 44 547-56

Zhu R Y, Stone H, Newman H, Zhou T Q, Tan H R and He C F 1991 A study on radiation damage in doped BGO crystals Nucl. Instrum. Methods Phys. Res. A 302 69-75 